\title{
Alterações patológicas causadas por nematóides parasitas de jararaca (Bothrops jararaca Wied, 1824) criadas em cativeiro
}

\section{Pathological aspects of nematodes of "jararaca" (Bothrops jararaca Wied, 1824) reared in captivity}

\author{
Dalton Garcia de Mattos Junior, ${ }^{*}$ Eduardo C. Nogueira Rubião, ${ }^{* *}$ Rogério Tortelly, ${ }^{* * *}$ \\ Eliana de Fátima Marques de Mesquita, ${ }^{* * *}$ Rodrigo Caldas Menezes ${ }^{\star * * * *}$
}

\begin{abstract}
Resumo
Dezesseis exemplares de serpentes, denominadas vulgarmente jararacas (Bothrops jararaca), criadas em serpentário do Instituto Vital Brazil, Niterói, RJ, foram estudadas a fim de atestar a presença de parasitas nematóides. Lesões macroscópicas e microscópicas associadas dos órgãos parasitados foram observadas através de necropsia. Os nematóides Ophidascaris (Ascarididae), Kalicephalus (Diaphanocephalidae) e Rhabdias (Rhabdiasidae) foram encontrados em 14 (87,5\%) exemplares e a prevalências foi de 35,7\%, 35,7\% e 50\% para Ophidascaris, Kalicephalus e Rhabdias respectivamente. Ophidascaris travassoi Vaz, 1935, Kalicephalus costatus costatus Rud, 1819 e Rhabdias labiata Pereira, 1927 foram identificados. As lesões macroscópicas e microscópicas associadas causadas pelo Ophidascaris e Rhabdias foram os mais importantes achados de necropsias nestes hospedeiros.
\end{abstract}

Palavras-chave: Bothrops jararaca, nematóides, patologia.

\begin{abstract}
Sixteen snakes (Bothrops jararaca) reared at Instituto Vital Brazil, Niterói, Rio de Janeiro State were studied to identify the presence of nematodes parasites. Macroscopic and microscopic lesions of the parasitized organs were observed through necropsia. Nematodes Ophidascaris (Ascarididae), Kalicephalus (Diaphanocephalidae) and Rhabdias (Rhabdiasidae) were found out in 14 (87,5\%) snakes and the prevalence were $35,7 \%, 35,7 \%$ and $50 \%$ to Ophidascaris, Kalicephalus and Rhabdias respectivelly. Ophidascaris travassoi Vaz, 1935; Kalicephalus costatus costatus Rud, 1819 and Rhabdias labiata Pereira, 1927 were identified. The macroscopic and microscopic lesions caused by Ophidascaris and Rhabdias were the most important findings in these hosts.
\end{abstract}

Keywords: Bothrops jararaca, nematodes, pathology.

\section{Introdução}

A família Viperidae inclui as serpentes peçonhentas mais importantes sob o ponto de vista médico e veterinário, com os gêneros Porthidium, Bothriopsis, Lachesis, Crotalus e Bothrops (Campbell, 1989).

As serpentes do gênero Bothrops, conhecidas como jararacas, são muito abundantes e amplamente distribuídas no Brasil, sendo responsáveis por numerosos acidentes ofídicos no homem e nos animais (Sorensen, 1990).

A criação de jararacas em cativeiro tem como finalidade principal a extração da peçonha, matéria-prima para a produção de soros antiofídicos e na elaboração de outros fármacos.
Esta atividade é considerada de grande importância para a segurança do homem e dos animais que vivem nas zonas rurais expostos aos acidentes ofídicos.

Problemas de parasitoses que acometem jararacas em cativeiro são relativamente comuns e os nematóides desempenham um papel marcante e causam enfermidades que acarretam elevada mortalidade entre os indivíduos. Estes problemas têm sido relatados por diversos especialistas em todo o mundo (Fiennes,1961 ; Fowler, 1986; Frye, 1991), porém, no Brasil, as informações ainda são muito escassas, relacionando-se, principalmente à descrição e taxonomia de helmintos (Freitas, 1968; Fábio e Rolas, 1974; Rodrigues et al., 1990; Vicente et al., 1993).

* Professor do Departamento de Saúde Coletiva Veterinária e Saúde Pública da Faculdade de Veterinária da Universidade Federal Fluminense. Rua Dr. Vital Brazil Filho, 64, Niterói, RJ, - CEP 24230-340. E-mail: dgmjrvet@vm.uff.br

** Acadêmico do Curso de Pós-Graduação em Medicina Veterinária - Área de Clínica Médica da Faculdade de Veterinária da Universidade Federal Fluminense, Niterói, RJ.

*** Professor do Departamento de Patologia do Centro de Ciências Médicas da Universidade Federal Fluminense, Niterói, RJ.

**** Professor do Departamento de Tecnologia dos Alimentos da Faculdade de Veterinária da Universidade Federal Fluminense, Niterói, RJ.

***** Médico-veterinário do CECAL-FIOCRUZ, Rio de Janeiro, RJ. 
A presente pesquisa tem como objetivo estudar a ocorrência e descrever as lesões determinadas pelos principais nematóides de jararacas criadas no serpentário do Instituto Vital Brazil.

\section{Material e métodos}

Foram examinados 16 exemplares de serpentes da espécie Bothrops jararaca (jararaca) do plantel do serpentário do Instituto Vital Brazil, localizado em Niterói, RJ. Os animais foram acompanhados, por um período de oito meses, a fim de se observar sinais clínicos sugestivos de enfermidades.

Cada animal encontrado doente era submetido a tratamento. Caso ocorresse o óbito, procedia-se à necropsia, de acordo com as técnicas usuais em helmintologia. Uma ficha de identificação individual foi confeccionada para que as informações fossem anotadas, desde a procedência do animal até os exames diagnósticos definitivos.

Procedeu-se a observação macroscópica dos órgãos internos assim como a presença de lesões associadas aos nematóides. Fragmentos de tecidos foram fixados em formol à $10 \%$ e enviados ao Laboratório de Anatomia Patológica Veterinária da Universidade Federal Fluminense, a fim de serem processados de acordo com as técnicas histológicas (Behmer, 1976).

Os nematóides encontrados foram coletados, contados, fixados em AFA aquecido, preparados de acordo com Ueno e Gonçalves (1988) e, posteriormente, identificados de acordo com Yamaguti (1961) e Vicente et al. (1993).

\section{Resultados}

Dos 16 exemplares de serpentes examinados, 14 (87,5\%) apresentaram-se parasitadas. Os nematóides encontrados e identificados foram Ophidascaris travassoi Vaz, 1935 (Ascarididae) em cinco (35,7\%), Kalicephalus costatus costatus Rud, 1819 (Diaphanocephalidae) em cinco $(35,7 \%)$ e Rhabdias labiata Pereira, 1927 (Rhabdiasidae) em sete (50\%).

Das 11 fêmeas necropsiadas, nove $(81,8 \%)$ apresentaram-se parasitadas, sendo que quatro $(36,3 \%)$ demonstraram a presença de Rhabdias labiata, quatro (36,3\%) apresentaram-se infectadas por Ophidascaris travassoi e três $(27,2 \%)$ por Kalicephalus costatus costatus.

Os cinco machos necropsiados apresentavam-se parasitados por Rhabdias labiata em três casos, seguido de Kalicephalus costatus costatus com dois casos e Ophidascaris travassoi em apenas um caso.

Das 14 serpentes, duas (14,2\%) apresentaram infecções mistas, albergando espécimens representantes de mais de um gênero de nematóides.

As alterações macroscópicas observadas no estômago causadas por Ophidascaris travassoi se resumiram em úlceras que apresentavam os nematóides inseridos e atingindo as camadas mucosa e submucosa do órgão (Figura 1). Estas úlceras apresentavamse recentes ou cicatrizadas, observando-se um espessamento da parede do órgão.

$\mathrm{F}^{*}$ fêmea

$\mathrm{M}^{* *}$ macho

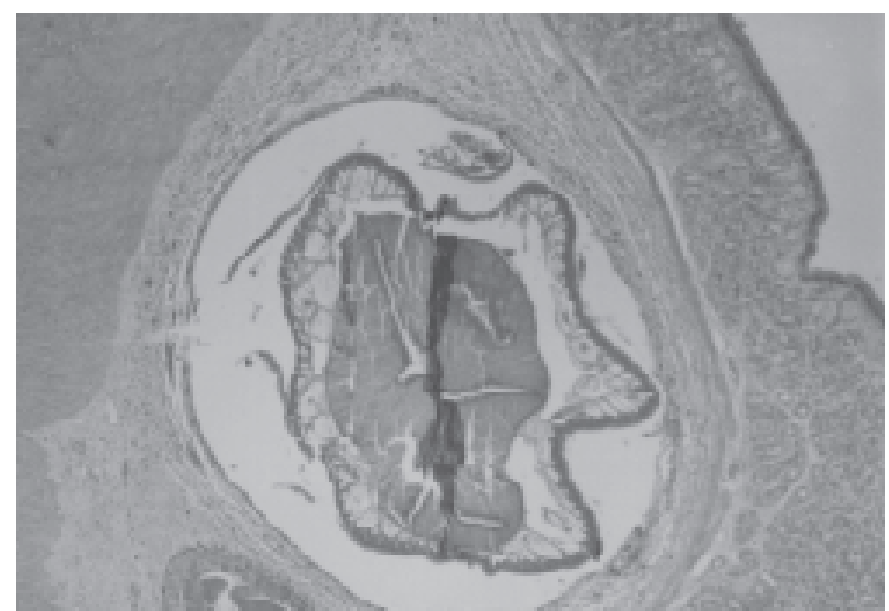

Figura 1: Estômago - Ophidascaris. Corte transversal na submucosa. $\mathrm{H}$ $x E-40 X$.

As alterações microscópicas determinadas por Ophidascaris travassoi eram representadas por granulomas de diferentes tamanhos, centralizados por áreas de necrose, característicos de migração parasitária, envoltos por uma forte reação inflamatória com presença de heterófilos. Alguns casos apresentavam úlceras que atingiam camadas da mucosa, submucosa e serosa, com forte reação inflamatória.

Espécimens de Kalicephalus costatus costatus foram observados entre as vilosidades intestinais, sem contudo estar associado a lesões macroscópicas visíveis nos sítios de encontro do helminto. Embora tenha sido observado um animal apresentando um quadro clínico de enterite, não foi possível associar a lesão com a presença do parasita. Não foram observadas alterações macroscópicas no intestino, em-

Tabela 1: Resultados das necropsias de 16 jararacas do plantel do serpentário do Instituto Vital Brazil, Niterói, RJ em 1998.

\begin{tabular}{lcrrccc}
\hline Número & sexo & \multicolumn{1}{c}{ compr. } & peso & Ophidascaris & Kalicephalus & Rhabdias \\
\hline B 1256 & $F^{*}$ & $875 \mathrm{~mm}$ & $80 \mathrm{~g}$ & 12 & - & - \\
B 1241 & $\mathrm{F}$ & $1110 \mathrm{~mm}$ & $220 \mathrm{~g}$ & - & 27 & - \\
B 1238 & $\mathrm{F}$ & $1150 \mathrm{~mm}$ & $165 \mathrm{~g}$ & 1 & - & - \\
B 1250 & $\mathrm{F}$ & $1280 \mathrm{~mm}$ & $549 \mathrm{~g}$ & 11 & - & - \\
B 1251 & $\mathrm{F}$ & $1164 \mathrm{~mm}$ & $695 \mathrm{~g}$ & - & - & 43 \\
B 1165 & $\mathrm{F}$ & $1150 \mathrm{~mm}$ & $245 \mathrm{~g}$ & - & 17 & - \\
B 393 & $\mathrm{F}$ & $730 \mathrm{~mm}$ & $105 \mathrm{~g}$ & - & - & - \\
B 1296 & M & $665 \mathrm{~mm}$ & $65 \mathrm{~g}$ & - & - & 12 \\
B 1300 & $\mathrm{F}$ & $1180 \mathrm{~mm}$ & $440 \mathrm{~g}$ & - & - & 3 \\
B 1301 & $\mathrm{F}$ & $1380 \mathrm{~mm}$ & $740 \mathrm{~g}$ & 7 & 10 & 12 \\
B 1207 & $\mathrm{M}$ & $830 \mathrm{~mm}$ & $55 \mathrm{~g}$ & - & 1 & - \\
B 732 & $\mathrm{F}$ & $1030 \mathrm{~mm}$ & $150 \mathrm{~g}$ & - & - & - \\
B 1278 & $\mathrm{F}$ & $950 \mathrm{~mm}$ & $73 \mathrm{~g}$ & - & - & 2 \\
B 1295 & $\mathrm{M}$ & $890 \mathrm{~mm}$ & $101 \mathrm{~g}$ & - & - & 17 \\
B 1282 & $\mathrm{M}$ & $795 \mathrm{~mm}$ & $50 \mathrm{~g}$ & - & - & 3 \\
B 1292 & $\mathrm{M}$ & $1000 \mathrm{~mm}$ & $249 \mathrm{~g}$ & 1 & 4 & - \\
Total 16 & $11 \mathrm{f} / 5 \mathrm{~m}$ & $665-1380 \mathrm{~mm}$ & $55-740 \mathrm{~g}$ & 32 & 59 & 92 \\
\hline
\end{tabular}


bora tenha sido registrada uma ligeira hiperemia da mucosa intestinal em algumas serpentes (Figura 2).

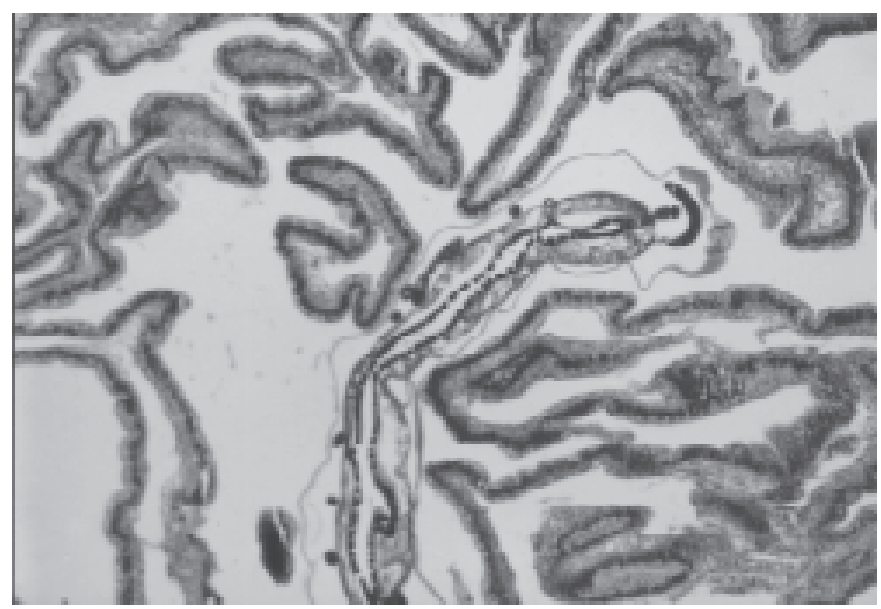

Figura 2: Intestino - Kalicephalus. Corte longitudinal em meio às vilosidades intestinais. $\mathrm{H} \times \mathrm{E}-40 \mathrm{X}$.

Espécimens de Rhabdias labiata foram encontrados nos pulmões de sete animais. Estes órgãos apresentavam focos múltiplos de um processo granulomatoso contendo em seu interior um material necrosado, restos de helmintos e heterófilos (Figura 3). As lesões por Rhabdias labiata determinaram um ligeiro aumento das secreções pulmonares e discreta reação inflamatória difusa com predomínio de heterófilos.

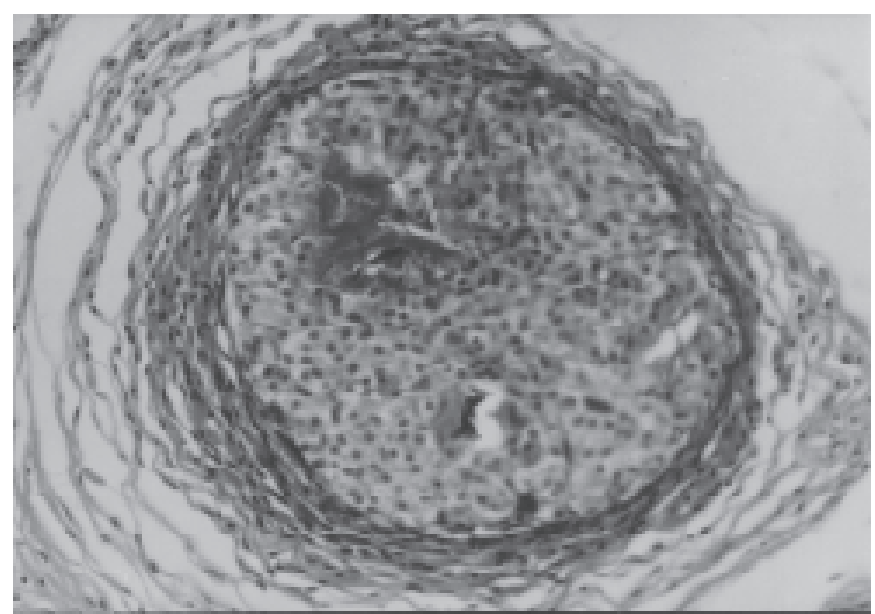

Figura 3: Pulmão demonstrando um granuloma epitelióide envolto por heterófilos e centralizado por fragmentos da cutícula de helminto.

\section{Referências}

BARNARD, S. M. Color Atlas of Reptilian Parasites. Veterinary Learning Systems Co. Inc. v. 8, 369 p., 1986.

BEHMER, O. A.; Castro de Tolosa, E. M.;FREITAS NETO, A. G. Manual de Técnicas para Histopatologia Normal e Patológica. São Paulo: Edart. Ed. da Universidade de São Paulo. 241 p. 1976.

CAMPBELL, J. A.; LAMAR, W. W. The Venomous Reptiles of Latin America. New York: Cornell University Press, 1989. 425 p.

FÁBIO, S.; Rolas, F. T. J. Sobre alguns helmintos parasitas de Dryadophis bifossatus (Raddi). Mem. Inst. Oswaldo Cruz, v. 72, n. 1, 2, p. 153, 1974.

\section{Discussão}

A ocorrência de nematóides Ophidascaris travassoi, Kalicephalus costatus costatus e Rhabdias labiata em diferentes espécies de serpentes foi observada por diversos investigadores (Fiennes,1961; Wallack,1983; Barnard, 1986; Fowler 1986; Troiano,1991 e Kolesnikovas, 1997). Dados sobre a prevalência destes nematóides em serpentários são escassos conforme cita Barnard (1986), mas pode-se considerar que a prevalência de $50 \%$ de Rhabdias labiata é alta, frente ao número de serpentes necropsiadas neste trabalho.

Existem poucos trabalhos científicos sobre nematóides de serpentes no Brasil e que não descrevem com detalhes as alterações macroscópicas e microscópicas determinadas por esses parasitas, especialmente em jararacas, nem o registro sobre a freqüência, prevalência de Ophidascaris travassoi no estômago, Kalicephalus costatus costatus no intestino delgado e Rhabdias labiata no pulmão. De acordo com resultados, estes nematóides foram comuns no serpentário, o que sugere que os parasitas são importantes nestes hospedeiros.

As lesões causadas por Ophidascaris travassoi estão de acordo com aquelas observadas por Fiennes (1961), Fowler (1986) e Frye (1991) em outras espécies de serpentes.

Embora ocorram relatos de enterites ulcerativas, hemorragias e obstruções intestinais causadas por Kalicephalus costatus costatus, no presente trabalho não foram observadas lesões que pudessem estar associadas aos parasitas, provavelmente diante do baixo grau de parasitismo encontrado nestes hospedeiros.

Nos pulmões parasitados por Rhabdias labiata observaramse alterações focais inflamatórias, representadas por granulomas centralizados por necrose e restos da cutícula dos nematóides. Estas lesões podem estar associadas às pneumonias graves com alta mortalidade de serpentes, conforme tem sido registrado por Wallak (1983). Diante dessas observações, constata-se que os nematóides Ophidascaris travassoi e Rhabdias labiata foram os mais importantes associados às enfermidades das serpentes criadas no serpentário do Instituto Vital Brazil.

\section{Agradecimentos}

Ao Dr. Melgarejo, chefe do serpentário do Instituto Vital Brazil, Niterói, RJ.

FIENNES, R. N. Diseases of Snakes Caused by Internal Parasites. London: The Zoological Society of London: 1961, Part I, 324 p.

FOWLER, M.E. Zoo and Wild Animal Medicine. $2^{\text {nd }}$ ed. Ed. Philadelphia W.B. Saunders Company, 1986. 1127 p.

FREITAS, J. F. T. Revisão do gênero Ophidascaris Baylis,1921 (Nematoda, Ascariodea). Mem. Inst. Oswaldo Cruz, v. 1, p. 129, 1968.

FRYE, F. L. Reptile Care and Atlas of Diseases and Treatments. v. 1, T.F.H. Publications Inc., 1991. 325 p.

KOLESNIKOVAS, C. K. M. Patologia comparada de cascavéis (Crotalus durissus Laurenti, 1768) mantidas em cativeiro. 1997.60 p. Tese (Mestrado)-Faculdade de Medicina Veterinária e Zootecnia da Universidade de São Paulo. 
RODRIGUES, H. O. et al. Contribution to the knowledge of the helminthological fauna of vertebrates of Maricá, Rio de Janeiro. Mem. Inst. Oswaldo Cruz, v. 85, n. 1, p. 116, 1990.

SOERENSEN, B. Animais peçonhentos. Rio de Janeiro: Atheneu, 1990. $138 \mathrm{p}$.

TROIANO, J. C. Manejo sanitário de reptiles en cativerio. Buenos Aires: Prensa Veterinaria Argentina, 1991.176 p.
UENO, H.; Gonçalves, P. C. Manual para o diagnóstico das helmintoses de ruminantes. 2. ed. Tokyo, Japan: JICA, 1988. 166 p.

VICENTE, J. J. Nematóides do Brasil. Parte III: Nematóides de Répteis. Revta bras. Zool. 168 p. 1993.

WALLACH, J. D. Diseases of Exotic Animals. Philadelphia: W.B Saunders Co, 1983, 1159 p.

YAMAGUTI, S. Systema Helminthum. Nematodes. Part I. New York: Interscience Publishers Inc, 1961.679 p. 